\title{
Fast Segmentation for Texture-based Cartography of whole Slide Images
}

\author{
Grégory Apou ${ }^{1}$, Benoît Naegel ${ }^{1}$, Germain Forestier ${ }^{2}$, Friedrich Feuerhake ${ }^{3}$ and Cédric Wemmert ${ }^{1}$ \\ ${ }^{1}$ ICube, University of Strasbourg, 300 bvd Sébastien Brant, 67412 Illkirch, France \\ ${ }^{2}$ MIPS, University of Haute Alsace, 12 rue des Frères Lumiere, 68093 Mulhouse, France \\ ${ }^{3}$ Institute for Pathology, Hannover Medical School, Carl-Neuberg-Straße 1, 30625 Hannover, Germany
}

\begin{abstract}
Keywords: Whole Slide Images, Biomedical Image Processing, Segmentation, Classification
Abstract: In recent years, new optical microscopes have been developed, providing very high spatial resolution images called Whole Slide Images (WSI). The fast and accurate display of such images for visual analysis by pathologists and the conventional automated analysis remain challenging, mainly due to the image size (sometimes billions of pixels) and the need to analyze certain image features at high resolution. To propose a decision support tool to help the pathologist interpret the information contained by the WSI, we present a new approach to establish an automatic cartography of WSI in reasonable time. The method is based on an original segmentation algorithm and on a supervised multiclass classification using a textural characterization of the regions computed by the segmentation. Application to breast cancer WSI shows promising results in terms of speed and quality.
\end{abstract}

\section{INTRODUCTION}

In recent years, the advent of digital microscopy deeply modified the way certain diagnostic tasks are performed. While the initial diagnostic assessment and the interpretation histopathological staining results remain a domain of highly qualified experts, digitization paved the way to semi-automated image analysis solutions for biomarker quantification and accuracy control. The emerging use of digital slide assessment in preclinical and clinical biomarker research indicates that the demand for image analysis of WSI will be growing. In addition, there is a rising demand in solutions to integrate multimodal data sets, e.g. complex immunohistochemistry (IHC) results with comprehensive genomic, or clinical data. With the expected increase of the number and quality of slide scanning devices, along with their great potential for use in clinical routine, increasingly huge amounts of complex image data (commonly called Whole Slide Images, WSI) will become available. Consequently, pathologists are facing the challenge to integrate complex sets of relevant information, partially based on conventional morphology, and partially on molecular genetics and computer-assisted readout of single immunohistochemistry (IHC) parameters (Gurcan et al., 2009).

However, there are still many challenges to the integration of WSI in routine diagnostic workflows in the clinical setting (Ghaznavi et al., 2013). Indeed, these images can contain hundreds of millions or even billions of pixels, causing practical difficulties for the storage, transmission, visualization and processing by conventional algorithms in a reasonable time. The Fig. 1 presents an example of a WSI of 18000 by 15000 pixels. Moreover, this new technology is still perceived as ineffective by pathologists who are more familiar with the use of classical light microscopy. Current drawbacks that still lead to relatively low acceptance of this technology include:

- variability in sample preparation (slice thickness, chemical treatments, presence of air bubbles and other unexpected elements) and in the acquisition process (lighting conditions, equipment quality);

- difficulties for human observers to comprehensively analyze quantitative readouts and subtle variations of intensity, requiring the use of heuristic methods for selecting relevant information, which introduces a risk of bias (Tavassoli and Devilee, 2003).

The field of WSI processing also follows the general trend in applied sciences of the proliferation of large databases generated by automated processes. Increasing quality and performance constraints imposed by modern medicine urge for the development of effective and automatic methods to extract information from these databases.

This paper presents a new approach, based on an object-oriented analysis (segmentation, classification)

This is the author's version of an article published at VISAPP 2014. The final authenticated version is available online at: https://doi.org/10.5220/ 0004687403090319 . 


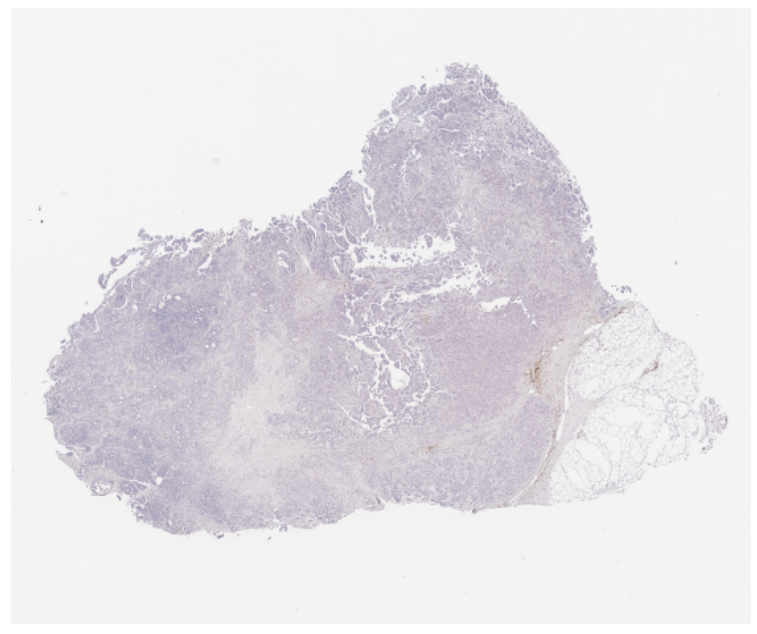

Figure 1: Example of Whole Slide Image.

to establish an automatic cartography of WSI. The main objective is to propose a decision support tool to help the pathologist to interpret the information contained by the WSI.

It is widely accepted that segmentation of a full gigapixel WSI is "impossible" due to time and memory constraints. We will show that it is in fact possible to segment such a large image in reasonable time with minimum memory requirements. Furthermore, the unexpected potential of simple color histogram to describe complex textures will be illustrated.

Compared to former works on WSI analysis, our contributions are: (i) an efficient computational framework enabling the processing of WSI in reasonable time, (ii) an efficient texture descriptor based on an automatic quantification of color histograms and (iii) a multiclass supervised classification based on expert annotations allowing a complete cartography of the WSI.

The paper is organized in 4 sections. First, existing approaches to analyze WSI are presented (Section 2), followed by the different steps of the method (Section 3). Then, experiments on WSI of breast cancer samples are described to evaluate the benefits of this approach (Section 4). Finally, we conclude and present some perspectives (Section 5).

\section{RELATED WORK}

As optical microscopy image analysis is a specific field of image analysis, a great variety of general techniques to extract or identify regions already exists. The main distinctive characteristic of the whole slide images (WSI) is their very large size, which makes impossible the application of number of conventional processing, despite of their potential interest.

Signolle and Plancoulaine (Signolle et al., 2008) use a multi-resolution approach based on the wavelet theory to identify the different biological components in the image, according to their texture. The main limitation of this approach is its speed: about 1 hour to analyze a sub-image of size $2048 \times 2048$ pixels, and several hundred hours for a complete image $(60000 \times$ 40000 pixels).

To overcome this drawback, several methods have been developed to avoid the need for analyzing entire images at full resolution. Thus, Huang et al. (Huang et al., 2010) noted that, to determine the histopathological grade of invasive ductal breast cancer using a medical scale called Nottingham Grading System (Elston and Ellis, 1991; Tavassoli and Devilee, 2003), it is important to detect areas of "nuclear pleomorphism" (i.e. areas presenting variability in the size and shapes of cells or their nuclei), but such detection is not possible at low resolution. So, they propose a hybrid method based on two steps: (i) the identification of regions of interest at low resolution, (ii) multiscale resolution algorithm to detect nuclear pleiomorphism in the regions of interest identified previously. In addition, through the use of GPU technology, it is possible to analyze a WSI in about 10 minutes, which is comparable to the time for a human pathologist.

Indeed, the same technology is used by Ruiz (Ruiz et al., 2007) to analyze an entire image $(50000 \times$ 50000 ) in a few dozen seconds by splitting the image into independent blocks. To manage even larger images (dozen of gigapixel) and perform more complex analyzes, Sertel (Sertel et al., 2009) uses a classifier that starts on low-resolution data, and only uses higher resolutions if the current resolution does not provide a satisfactory classification. In the same way, Roullier (Roullier et al., 2011) proposed a multiresolution segmentation method based on a model of the pathologist activity, starting from the coarsest to the finest resolution: each region of interest determined at one resolution is partitioned into 2 at the higher resolution, through a clustering performed in the color space. This unsupervised classification can be performed in about 30 minutes (without parallelism) on an image of size $45000 \times 30000$ pixels.

More recently, Homeyer (Homeyer et al., 2013) used supervised classification on tile-based multiscale texture descriptors to detect necrosis in gigapixel WSI in less than a minute. Their method shares some traits with our own, but we have a more straightforward workflow and use simpler texture descriptors on which we will elaborate more later.

The characteristics of our method compared to former ones are summarized in Table 1. 
Table 1: Comparison of our method with some existing ones. H\&E means Hematoxylin and Eosin, a widely used staining.

\begin{tabular}{|c|c|c|c|c|c|}
\hline Method & Pixels & Coloration & Classes & Performance & Parallelism \\
\hline (Ruiz et al., 2007) & $10^{9}$ & $\mathrm{H} \& \mathrm{E}$ & 2 (supervised) & $145 \mathrm{~s}$ (GeForce $7950 \mathrm{GX} 2)$ & GPU \\
\hline (Signolle et al., 2008) & $10^{9}$ & $\begin{array}{l}\text { Hematoxylin, } \\
\text { DAB }\end{array}$ & 5 (supervised) & $>100 \mathrm{~h}(\mathrm{Xeon} 3 \mathrm{GHz})$ & Unknown \\
\hline (Sertel et al., 2009) & $10^{10}$ & $\mathrm{H} \& \mathrm{E}$ & 2 (supervised) & $8 \mathrm{~min}($ Opteron $2.4 \mathrm{GHz})$ & $\begin{array}{l}\text { Cluster of } 8 \\
\text { nodes }\end{array}$ \\
\hline (Huang et al., 2010) & $10^{9}$ & $\mathrm{H} \& \mathrm{E}$ & $\begin{array}{l}4 \quad(\mathrm{ROI}+3 \\
\text { grades, super- } \\
\text { vised })\end{array}$ & $10 \min ($ GeForce $9400 \mathrm{M})$ & GPU \\
\hline (Roullier et al., 2011) & $10^{9}$ & $\mathrm{H} \& \mathrm{E}$ & 5 (unsupervised) & $30 \mathrm{~min}($ Core $2.4 \mathrm{GHz})$ & Parallelizable \\
\hline (Homeyer et al., 2013) & $10^{9}$ & $\mathrm{H} \& \mathrm{E}$ & 3 (supervised) & $\begin{array}{l}<1 \text { min (Core } 2 \text { Quad } 2.66 \\
\mathrm{GHz} \text { ) }\end{array}$ & Unknown \\
\hline Proposed method & $10^{8}$ & $\begin{array}{l}\text { Hematoxylin, } \\
\text { DAB + PRD }\end{array}$ & 6 (supervised) & 10 min (Opteron $2 \mathrm{GHz})$ & Parallelizable \\
\hline
\end{tabular}

\section{METHOD}

To achieve a fast and efficient classification of whole slide images, we propose a methodology enabling to partition the initial image in relevant regions. This approach is based on an original segmentation algorithm and on a supervised classification using a textural characterization of each region.

\subsection{Overview}

The proposed method relies on two successive steps: (i) image segmentation into segments or "patches"; (ii) supervised classification of these segments.

The main challenge of the segmentation step is to provide a relevant partioning of the image in an efficient way due to the large size of whole slide images. To cope with this problem, we propose to partition the image by using a set of horizontal and vertical optimal paths following image high gradient values.

The classification step is based on a textural approach where each region is labelled according to its texture description. This is justified by the fact that, to produce a decision, a pathologist analyzes the distribution of objects like cells, and the interaction between them rather than the objects themselves. We make the assumption that the distribution of objects can be described by a textural representation of a region.

A training set of texture descriptors is computed from a set of manually annotated images enabling a supervised classification based on a k-nearest neighbor strategy. The method overview is illustrated in Fig. 2 and Fig. 3.

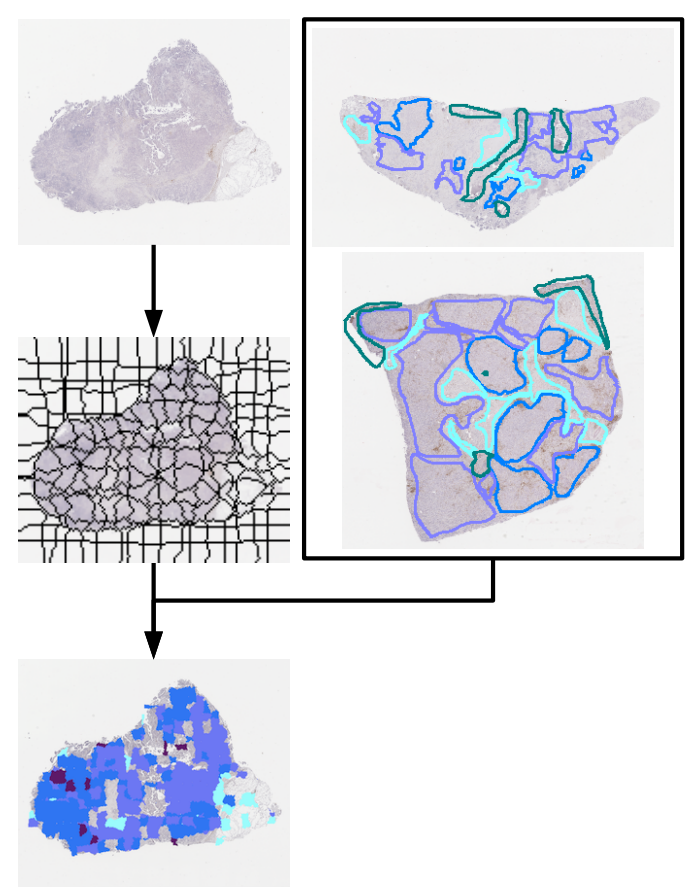

Figure 2: Method overview. Left: image under analysis, image partitioning and regions classification. Right: manually annotated images allowing the construction of the training set.

\subsection{Segmentation}

Let $f: E \rightarrow V$ be a 2D discrete color image defined over a domain $E \subseteq \mathbb{Z}^{2}$ with $V=[0,255]^{3}$. Let $f_{i}$ denotes the scalar image resulting of the projection of $f$ on its $i^{t h}$ band. We suppose that $E$ is endowed with an adjacency relation. A path is a sequence of points $\left(p_{1}, p_{2}, \ldots, p_{n}\right)$ such that, for all $i \in[1, \ldots, n-1], p_{i}$ 


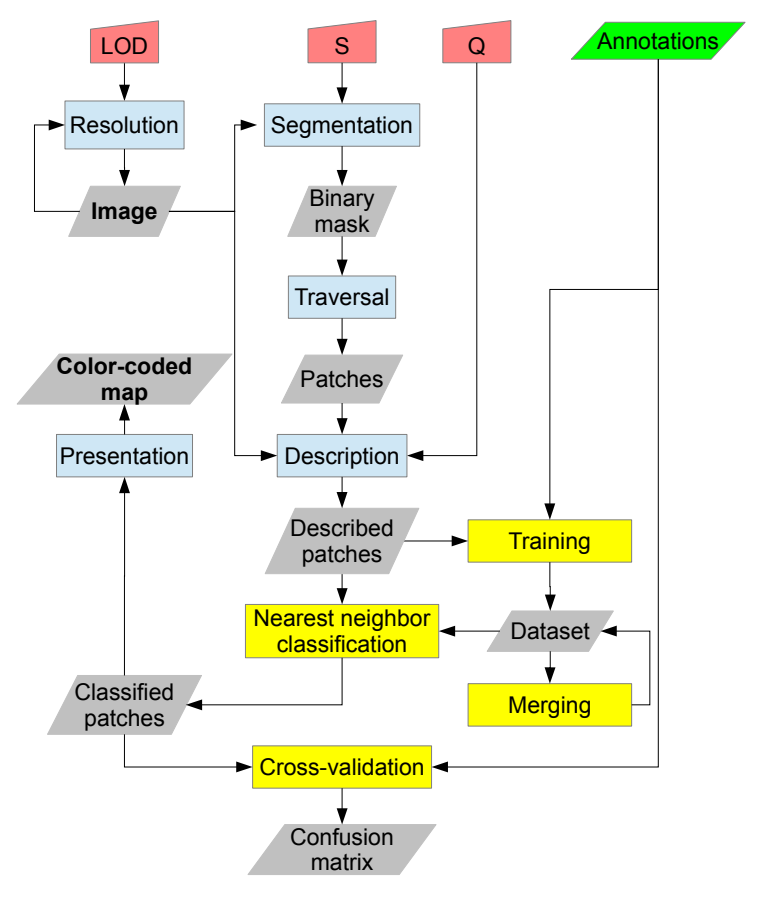

Figure 3: Data structures and processes. Red boxes represent parameters, green box represents expert annotations (ground truth). Blue boxes are image-related operations, yellow boxes are data mining-related. Gray boxes are data structures input, exchanged, modified and output by the processes.

and $p_{i+1}$ are adjacent points. Let $W, H$ be respectively the width and the height of $f$. The segmentation method is based on two successive steps.

First, the image $f$ is partitioned into $W / S$ vertical and $H / S$ horizontal strips, with $S>1$ an integer controlling the width of a strip.

Second, a path of optimal cost is computed from one extremity of the strip to the other in each image strip. The cost function is related to the local variations, hence favoring the optimal path to follow high variations of the image. More precisely, the local variation of $f$ in the neighborhood of $p$ is computed as:

$$
g(p)=\max _{q \in N(p)} d(f(p), f(q)),
$$

where $d$ is a color distance, and $N(p)$ the set of points adjacent to $p$. In our experiments we used $d(a, b)=\max _{i}\left|a_{i}-b_{i}\right|\left(L_{\infty}\right.$ norm $)$ and $N(p)=\{q \mid$ $\left.\|p-q\|_{\infty} \leq 1\right\}$ (8-adjacency).

The global cost associated to a path $\left(p_{i}\right)_{i \in[1 \ldots n]}$ of length $n$ is defined as:

$$
G=\sum_{i=1}^{n} g\left(p_{i}\right)
$$

From an algorithmic point of view, an optimal path maximising this summation can be retrieved us- ing dynamic programming (Montanari, 1971) in linear time with respect to the number of points in the strip, hence requiring to scan all image pixels at least once. To speed up the process, a suboptimal solution is computed instead by using a greedy algorithm: starting from an arbitrary seed at an extremity of the strip, the successive points of the path are added by choosing, in a local neighborhood, the point $q$ where $g(q)$ is maximal. By doing so, the values of $g$ are computed on the fly, only for the pixels neighboring the resulting path.

Some notable properties of this algorithm are: (i) its speed due to the fact that not all pixels need to be processed, (ii) a low memory usage even for large images because the only required structures are the current strip and a binary mask to store the result, (iii) its potential for parallelization, because all strips in a given direction can be processed independently.

Fig. 4 illustrates the steps of the segmentation method and Fig. 5 gives an example of the end result.

\subsection{Training}

To create a training base, the reference images are segmented into patches. Using the expert annotations, each patch is associated with a class or label. Then, by computing a texture descriptor for each patch, we can create an association between a texture and a group of labels. A texture can have several labels if it is present in regions of different classes. As a result, the training base can be modeled as a function $B: T \rightarrow G$ where $T$ is the set of texture descriptors and $G=\mathcal{P}(L)$ is the power set of all labels $L$.

When $|B(t)| \neq 1$, the texture $t$ is ambiguous. Section 4.2 describes how to measure this phenomenon and thus quantify the validity of the model. In order to perform the classification, all $B(t)$ must be singletons. To that end, $B$ is updated so that ambiguous textures are classified as excluded elements.

\subsection{Classification}

Some authors use distributions of descriptors to describe textures (Ojala et al., 1996). The chosen descriptors can be arbitrarily complex, and, as a starting point, we decided to use simple color histograms that are functions $H: V \rightarrow[0,1]$ that associate each pixel color to its frequency in a given patch. Given the fact that all images were obtained using the same process and equipment with the same settings, no image preprocessing was deemed necessary.

In order to perform a supervised multi-class classification, we opted for a one nearest neighbor classification because of its simplicity (no assumption 


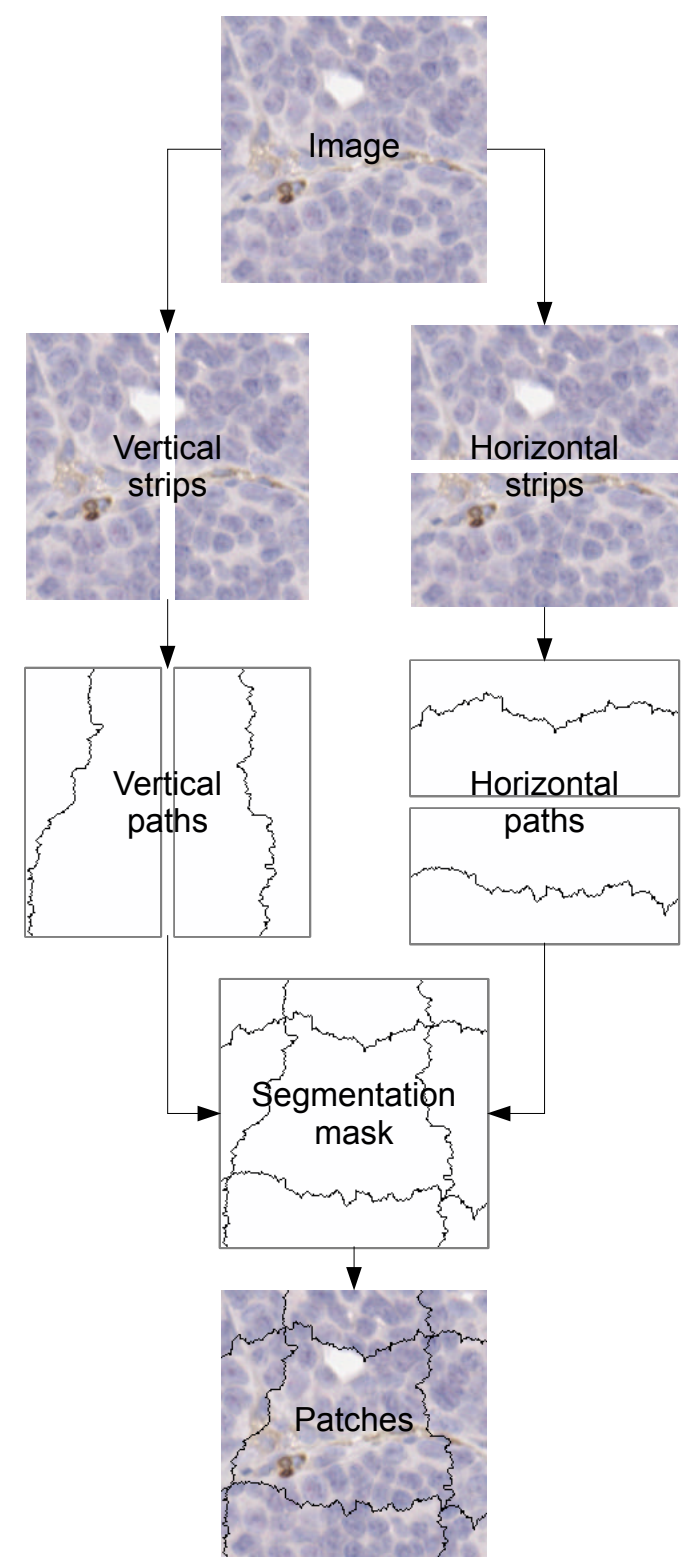

Figure 4: Path computation in horizontal and vertical strips, leading to an image partition.

needs to be made on the distribution of the textures in the descriptor space) and the sufficient amount of training samples available (which turned out to be a bit excessive with sometimes up to several millions of elements). For this kind of classification, we measure the distance between histograms using the euclidean metric:

$$
d\left(h_{1}, h_{2}\right)=\sqrt{\sum_{v}\left(h_{2}(v)-h_{1}(v)\right)^{2}}
$$

This choice of descriptor and metric is arbitrary and

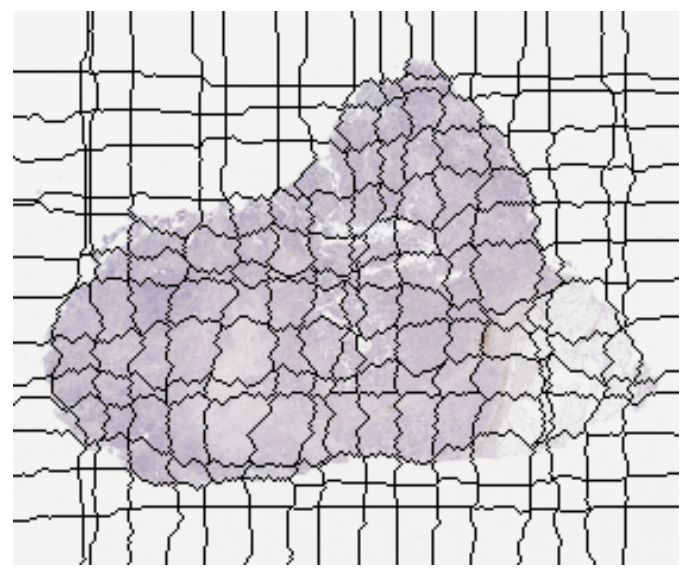

Figure 5: Example of image partitioning based on our algorithm.

will serve as reference for future work.

\section{EXPERIMENTS AND RESULTS}

\subsection{Data}

Unlike most of the work published on the subject, our images are obtained using a double-staining process (Wemmert et al., 2013). More precisely, we used formalin-fixed paraffin-embedded breast cancer

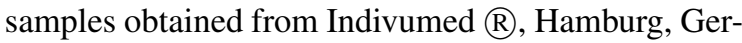
many. Manual immunohistochemistry staining was performed for CD8 or CD3/Perforin, and antibody binding was visualized using 3,3-diaminobenzidine tetrahydrochloride (DAB, Dako, Hamburg, Germany) and Permanent Red (PRD, Zytomed, Berlin, Germany). Cell nuclei were counterstained with hematoxylin before mounting. As a result, cancerous (large) and noncancerous (small) cell nuclei appear blue (hematoxylin), except for lymphocytes which can be appear red (PRD) and/or brown (DAB) depending on their state.

For our experiments, 7 whole slide images ranging from $1 \cdot 10^{8}$ to $5 \cdot 10^{8}$ pixels have been annotated by a pathologist using 6 labels: invasive tumor (solid formations), Fig. 6; invasive tumor (diffusely infiltrating pre-existing tissues), Fig. 7; intersecting stromal bands, Fig. 8; DCIS (Ductal Carcinoma In Situ) and invasive tumor inside ductal structures, Fig. 9; nonneoplastic glands and ducts, Fig. 10; edges and artifacts to be excluded, Fig. 11.

The annotations do not explicitly provide quantifiable cell characteristics that could be used to design a medically relevant cell-based region identification. Instead, they take the form of outlines that may or 


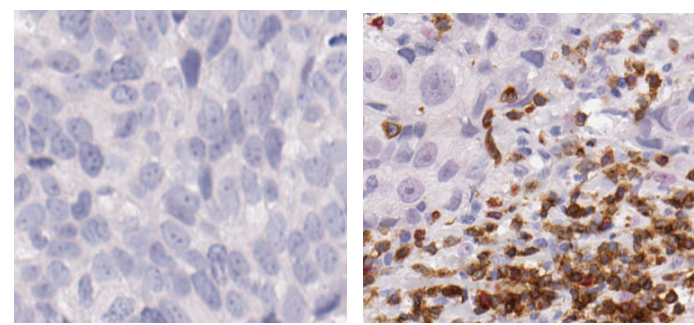

Figure 6: Annotation: Invasive tumor (solid formations). Description: High concentration of cancerous cells.

Note: All classes can contain foreign objects as can be seen on the right, and sometimes the same objects (foreign or not) can be seen in several classes (compare with Fig. 7).
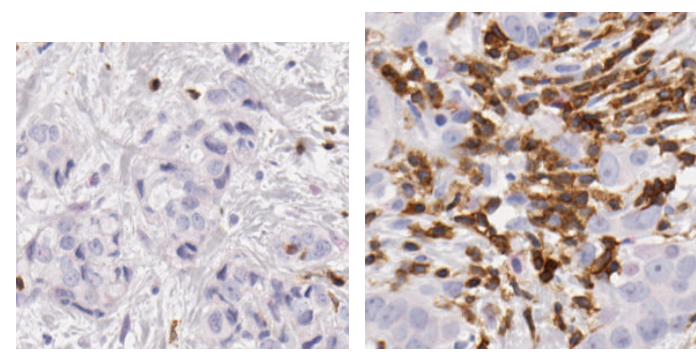

Figure 7: Annotation: Invasive tumor (diffusely infiltrating pre-existing tissues).

Description: Cancerous cells disseminated in noncancerous tissue.
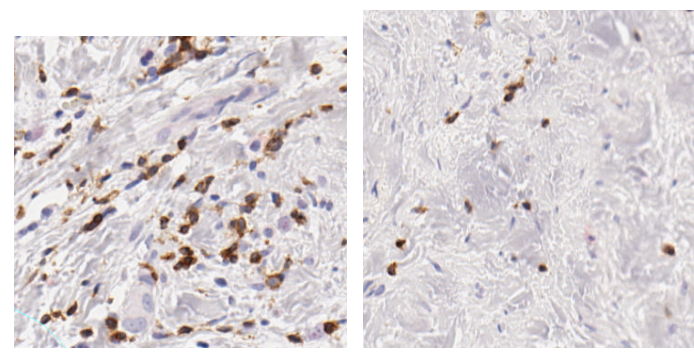

Figure 8: Annotation: Intersecting stromal bands. Description: Connective tissue.

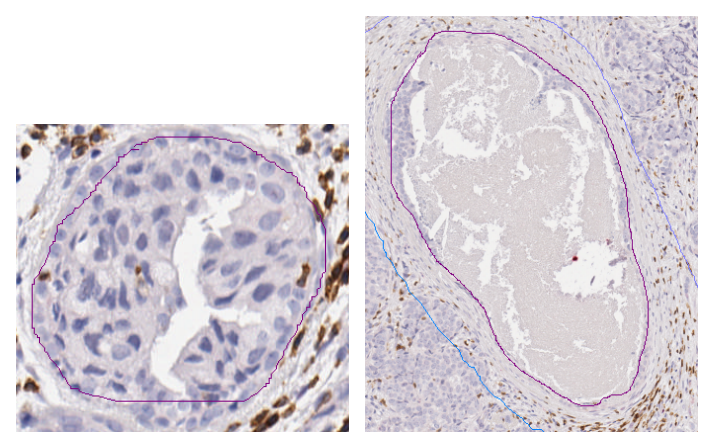

Figure 9: Annotation: DCIS and invasion inside ductal structures.

Description: A Ductal Carcinoma In Situ refers to cancer cells within the milk ducts of the breast.

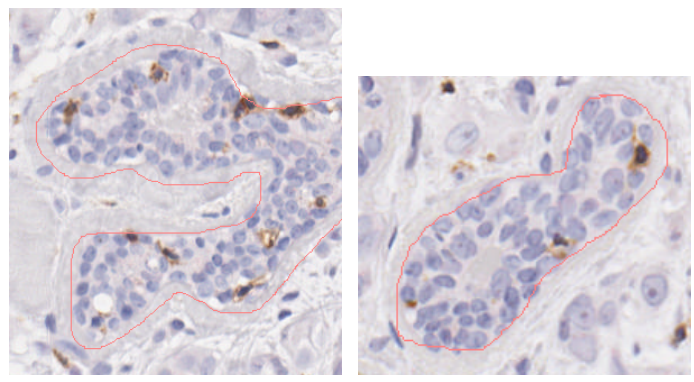

Figure 10: Annotation: Nonneoplastic glands and duct. Description: Noncancerous structures.
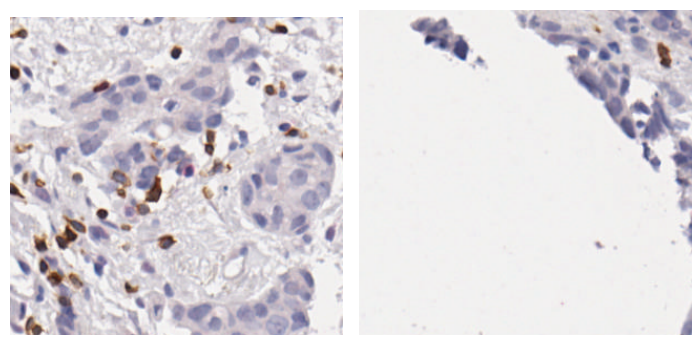

Figure 11: Annotation: Edges and artefacts to be excluded. Description: Nonbiological features (background, smudges, bubbles, blurry regions, technician's hair, ...) and damaged biological features (borders, defective coloration, missing parts, ...).

may not match visual features (Fig. 12). Even though some of these seem obvious, like the background, it is not easy for an untrained eye to establish a set of intuitive rules that would explain the expert's opinion, even after trying some simple visual filters (quantization, thresholding). Moreover, the classes are not uniformly represented in our data (Fig. 13): while excluded elements are described by only a handful of annotations, they actually account for the majority of the area of the images, especially because of the background; on the other hand, ductal structures constitute a minority and are sometimes completely missing.

Nonetheless, the delimited regions appear to exhibit a texture-related behavior, and we can use that to decide on a model: a delimited region is made of a set of patches that can be identified by their texture, and delimited regions of the same class share the same set of textures. Thus, by partitioning the image into patches and labeling each patch based on its texture, we can draw a color-coded map like in Fig. 14.

We already expected that some classes (ductal structures) would be difficult to distinguish with only texture information. But if the image can be decomposed into identifiable blocks, then it might be possible in future work to perform morphology-based analysis without the need for precise cell segmentation, giving a general framework to describe the contents of a histopathological image, regardless of the cell con- 


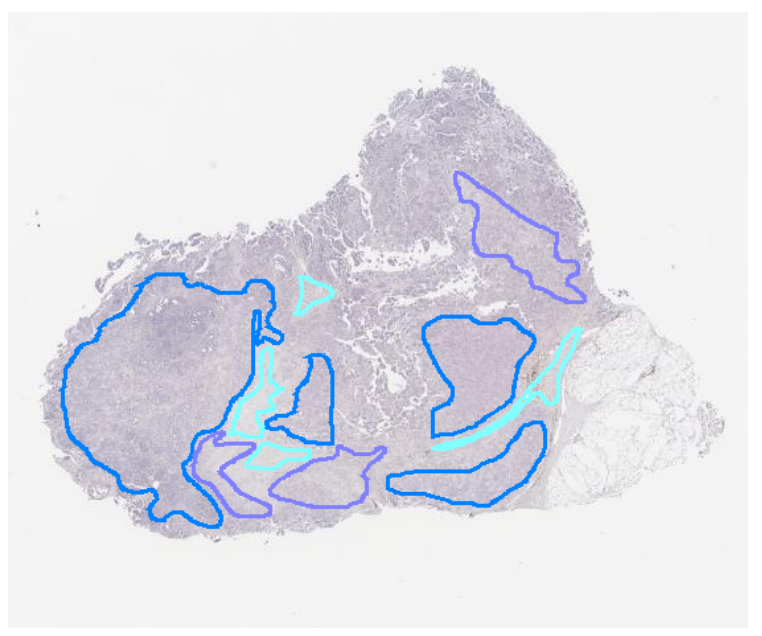

Figure 12: Expert annotations; the regions are outlined, each color represents a class; excluded regions (dark green) are given as examples, which is why the image is not fully annotated.

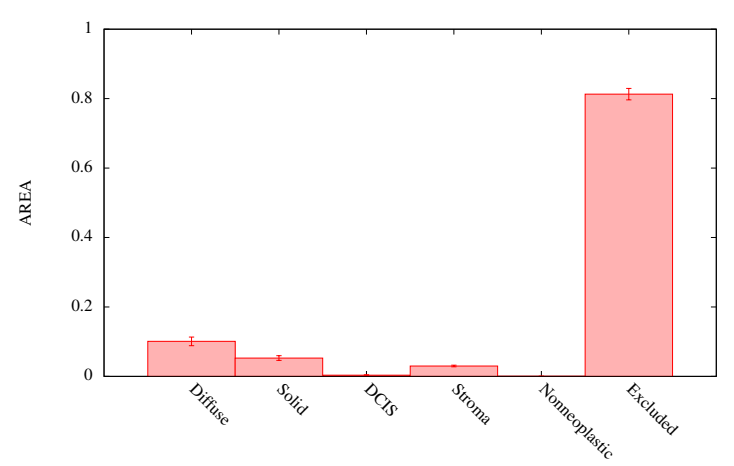

Figure 13: Relative areas of all the classes: average for each training set. Standard deviation is given as vertical bars.

tents of the studied classes.

\subsection{Model Evaluation}

The method is evaluated with a leave-one-out crossvalidation involving all the annotated images: for each image (in our set of 7), a training base is created with the other 6 . All the values given in the rest of this article are obtained by averaging the values from 7 experiments.

The quality of the model can be measured by computing the certainty of the training base for each label $l$ :

$$
C(l)=\frac{\left|B^{-1}(\{l\})\right|}{|\{t \in T: l \in B(t)\}|}
$$

When the certainty is $100 \%$, it means that the only group containing the label is a singleton, and so the textures can be used to uniquely characterize the cor-

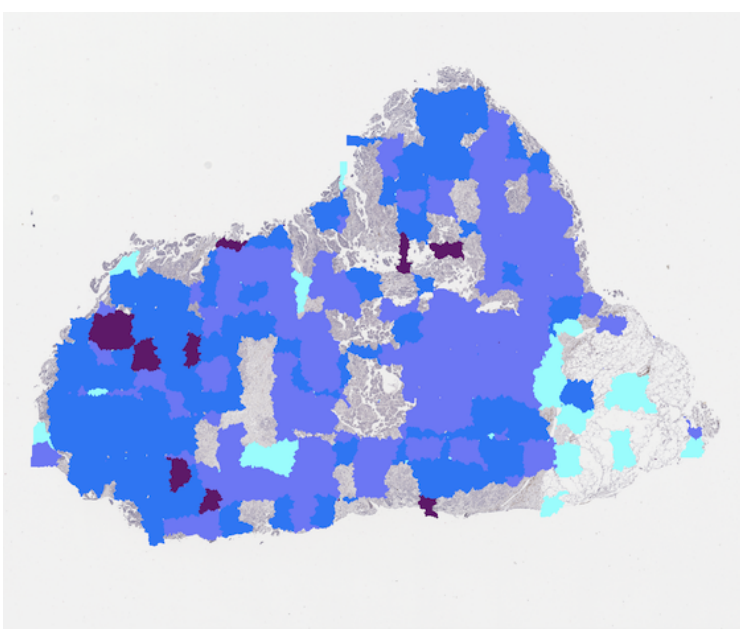

Figure 14: Classification map obtained by the presented method; the colors match those used by the expert, except for the excluded regions which are left untouched.

responding class. On the other hand, a certainty of $0 \%$ means that the textures are too ambiguous for a one-to-one mapping.

Since a human pathologist uses a multi-resolution approach (Roullier et al., 2011), a whole slide image is typically provided as a set of images corresponding to different magnifications that can be used by visualization software to speed up display. But they restrict the systematic study of the impact of the resolution level, and can also cause additional degradation due to lossy compression. So, in order to determine the information available at each level of detail (LOD), we compute for each image $I$ a pyramid defined by:

$$
I_{L O D}(x, y)=\frac{1}{4} \sum_{(i, j) \in\{0,1\}^{2}} I_{L O D-1}(2 x+i, 2 y+j)
$$

The original image is at LOD 0 (Fig. 15). It can be observed that high resolution is correlated with high data set certainty for the chosen texture descriptor (Fig. 16).

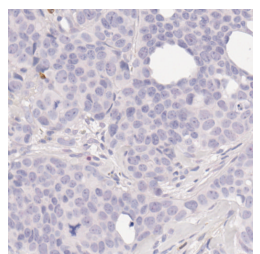

(a) LOD 0

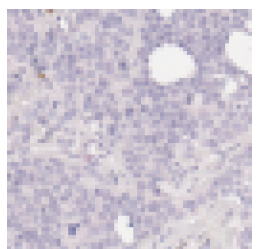

(b) LOD 3

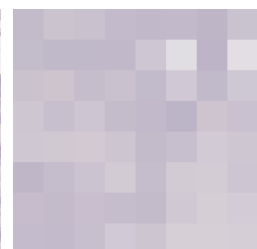

(c) LOD 6
Figure 15: Visualization of the effect of the resolution parameter LOD on pixel data.

Ideally, the segmentation algorithm should create patches of the right size, so that each patch would contain just enough information to identify a class- 


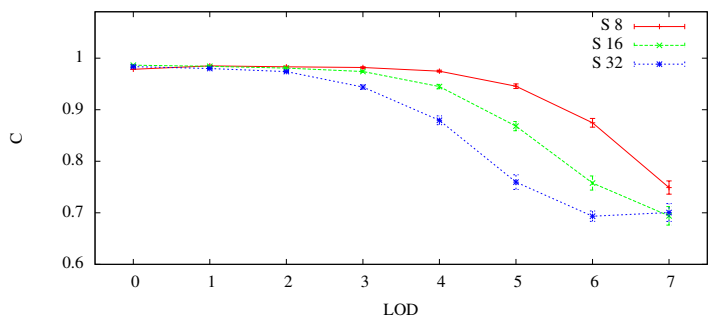

Figure 16: Visualization of the effect of the resolution parameter LOD on the overall certainty of the training base for different values of $S$ and $Q=4$. Standard deviation is given as vertical bars.

characteristic texture. Instead, we will assume the existence of a common texture scale that applies to all classes: the segmentation parameter S (Fig. 17). At high resolution, a texture described by its color can help identify a class with very little doubt (Fig. 16). But at lower resolutions, larger values of $\mathrm{S}$ increase the ambiguity of the texture description, because the patches become large enough to contain multiple textures from adjacent regions of different classes.

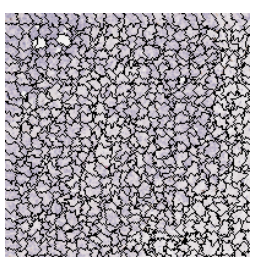

(a) $\mathrm{S} 8$

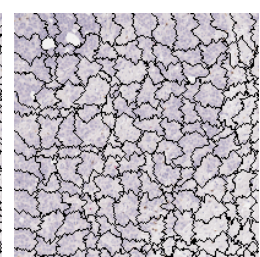

(b) $\mathrm{S} 16$

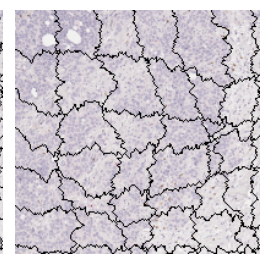

(c) S 32
Figure 17: Visualization of the effect of the segmentation parameter $\mathrm{S}$ on patch generation.

Finally, despite the use of sparse structures, a one nearest neighbor classification using color histograms requires large amounts of memory and processing time. A simple yet effective technique to mitigate this issue is to use a quantization scheme where the values used as histogram keys are $2^{Q}\left\lfloor\frac{v}{2 Q}\right\rfloor$ instead of $v$, and $Q$ is the quantization parameter. Less memory is required because textures with close descriptors are merged. As Fig. 18 shows, a mild quantization $(Q \leq 4)$ barely affects the certainty of the training base.

By considering only this measure of the training base quality, we would expect to get the best results with high resolution, small patches and minimal quantification. But we experimentally determined that the configuration (LOD 4, Q 4, S 32) yielded the best overall outcome when plotting the data in ROC space (Fig. 19). The discrepancy between high training base certainty and lesser classification results can

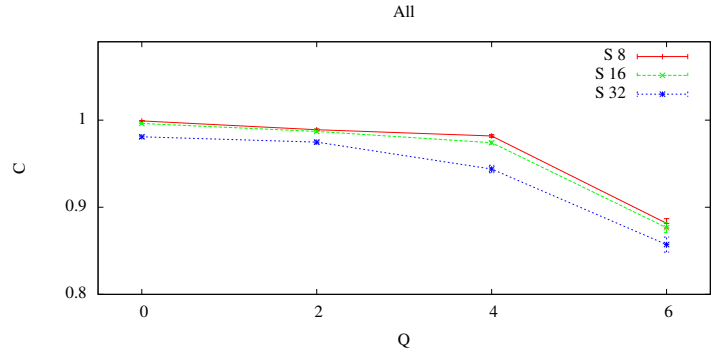

Figure 18: Visualization of the effect of the quantization parameter Q on the overall certainty of the training base for different values of $S$ and $L O D=4$. Standard deviation is given as vertical bars (barely visible because they are small).

have several causes, explored in the next section.

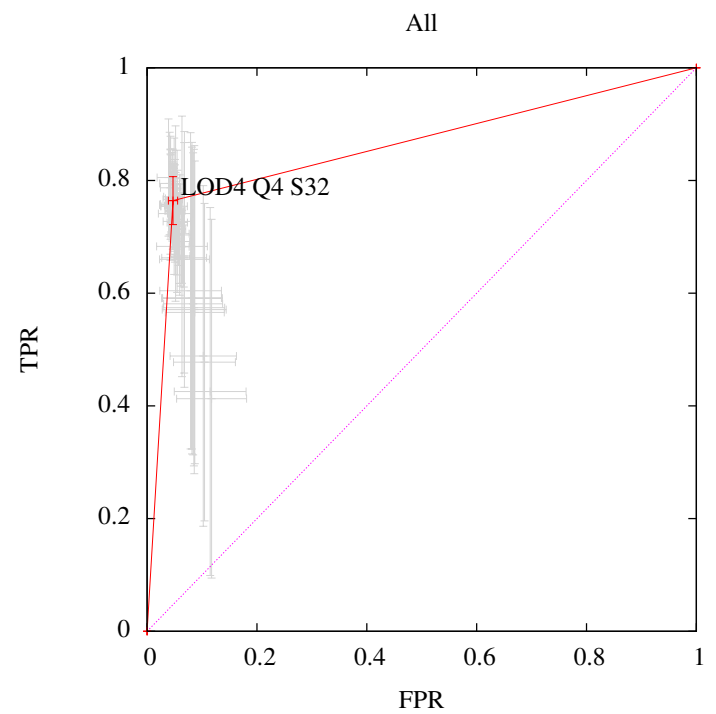

Figure 19: ROC points (light gray) for each parameterization are obtained by merging experimental data points for all the classes. For clarity, a convex curve (red) has been synthesized from these points with one point that stands out; such a synthetic curve makes sense because a classifier can be built for any interpolated point (Fawcett, 2006). Standard deviation is given as horizontal and vertical bars.

\subsection{Classification results}

By observing the confusion matrix for a chosen set of parameters (table 2), we can see that the class of the excluded regions is the only one to be adequately detected.

The ductal structures of the DCIS class are difficult to identify because of their ambiguous textures, but also because of their rarity. Surprisingly, the non- 
neoplastic ductal structures seem more prone to textural characterization, but the values are inconclusive due to the high standard deviation caused by the rarity of the class and the small number of annotated images.

The remaining 3 classes illustrate some limitations of the model. The "stroma" class is detected as "excluded regions", "stroma" and "diffuse invasion". As it turns out, "diffuse invasion" means that textures corresponding to cancerous cells are mixed with textures corresponding to stroma. This mixing creates conflicts which are resolved by assigning the "excluded regions" class to the ambiguous textures (section 3.3). The same phenomenon explains why both "solid formations" (regions of high cancerous cell density) and "diffuse invasion" (regions of low cancerous cell density) are detected as a mixture of "excluded regions", "solid formations" and "diffuse invasion". The pathologist who made the annotations told us that the difference between "solid formation" and "diffuse invasion" can be debatable. So, by merging these 2 classes, we could get a much higher detection rate (possibly up to $90 \%$ ).

But the underlying problem is that the model is flawed: a texture unit defined by one patch is not enough to identify a class. The certainty of the training bases is high because, unexpectedly, simple color histogram are not only strong enough to describe such texture units, but they also capture small variations that can almost identify the patches themselves, hence the large size of the training bases (see next section). The images are mostly "clean", the only visually (barely) noticeable noise seeming to be compression artifacts (lossy JPEG2000). So these variations are more likely a "content noise", a mix of a variety of biological and nonbiological elements. When used to analyze a new image, these small variations give way to the main constituents of the texture. We conjecture that if we could remove these variations, we would obtain a small set of textures (maybe a few dozens) that could be used to identify regions like a pathologist does. With that in mind, the confusion matrix could suggest that regions corresponding to stroma, diffuse invasion and solid formations are made up of the same set of textures (as described by color histograms of patches), but they differ by the proportions of these textures; that phenomenon could be quantified by measuring their local density distributions.

Another clue in support of that conjecture is the specificity data (table 3 ). While the presence of a given texture is not always enough to identify only one given class, its presence might still be a necessity and thus its absence can reliably be used to exclude some classes, especially for stroma (sparsely popu- lated regions with a "sinuous" appearance) and solid formations (dense clumps of large cancerous cell nuclei). The specificity for the excluded regions is lower because of its role as "default class" to resolve ambiguities in our current method, as explained previously. At this time, we believe that we don't have enough data on DCIS and nonneoplastic objects to draw a definite conclusion on these classes.

\subsection{Performance}

The experiments were run on an AMD Opteron 2 $\mathrm{GHz}$ with $32 \mathrm{~Gb}$ of memory. The segmentation step takes at most a few minutes even on large images (less than 2 minutes for a full gigapixel image with our current sequential Java implementation). But, as shown on Fig. 20, the main bottleneck of the method is the size of the training bases, mostly because of the time needed to search a nearest neighbor. So far, this has prevented us from testing our current algorithm with high resolutions but we have verified that capping the training base size to 10000 elements (which is still large) can bring down the computing time to less than 2 hours for the highest resolution. That being said, our current results suggest that lower resolutions may already have enough information to completely analyze the image.

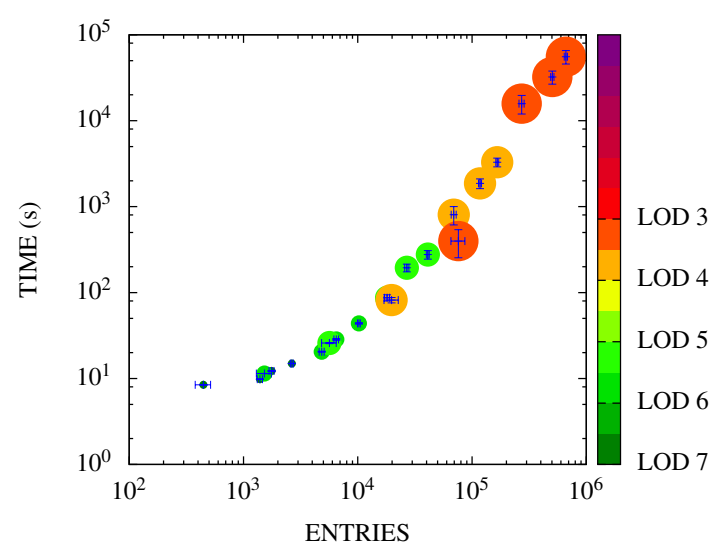

Figure 20: Mean sequential calculation time by image according to the size of the training base. Each point corresponds to a setting (LOD, Q) for a segmentation of size 32 pixels. The colored discs symbolize the image size, related to the parameter LOD. Some experiments at high resolution were not performed because of excessive time and memory requirements. Standard deviation is given as horizontal and vertical bars. 
Table 2: Confusion matrix for a particular parameterization (LOD 4, Q 4, S 32). Results are given as mean and standard deviation computed from 7 values.

\begin{tabular}{c|cccccc} 
& DCIS & $\begin{array}{c}\text { Excluded } \\
\text { regions }\end{array}$ & Stroma & $\begin{array}{c}\text { Solid } \\
\text { formations }\end{array}$ & $\begin{array}{c}\text { Nonneoplastic } \\
\text { objects }\end{array}$ & $\begin{array}{c}\text { Diffuse } \\
\text { invasion }\end{array}$ \\
\hline DCIS & $\mathbf{5 7 \%} \pm \mathbf{4 9}$ & $29 \% \pm 35$ & $2 \% \pm 5$ & $4 \% \pm 4$ & $0.1 \% \pm 0.2$ & $8 \% \pm 10$ \\
$\begin{array}{c}\text { Excluded } \\
\text { regions }\end{array}$ & $0.3 \% \pm 0.4$ & $\mathbf{8 5} \% \pm \mathbf{3}$ & $3 \% \pm 1$ & $3 \% \pm 2$ & $0.2 \% \pm 0.2$ & $9 \% \pm 3$ \\
Stroma & $1 \% \pm 3$ & $29 \% \pm 10$ & $\mathbf{3 1} \% \pm \mathbf{1 0}$ & $4 \% \pm 3$ & $0.1 \% \pm 0.2$ & $25 \% \pm 10$ \\
$\begin{array}{c}\text { Solid } \\
\text { formations }\end{array}$ & $0.9 \% \pm 2$ & $19 \% \pm 14$ & $0.5 \% \pm 0.6$ & $\mathbf{4 4} \% \pm \mathbf{3 2}$ & $0.0 \% \pm 0.1$ & $35 \% \pm 24$ \\
$\begin{array}{c}\text { Nonneoplastic } \\
\text { objects }\end{array}$ & $0 \% \pm 0$ & $11 \% \pm 20$ & $0.6 \% \pm 1$ & $6 \% \pm 14$ & $\mathbf{7 1} \% \pm \mathbf{4 5}$ & $12 \% \pm 18$ \\
$\begin{array}{c}\text { Diffuse } \\
\text { invasion }\end{array}$ & $0.2 \% \pm 0.2$ & $37 \% \pm 12$ & $3 \% \pm 3$ & $11 \% \pm 9$ & $2 \% \pm 3$ & $\mathbf{4 7 \%} \% \mathbf{1 5}$
\end{tabular}

Table 3: Sensitivity and specificity for a particular parameterization (LOD 4, Q 4, S 32). Results are given as mean and standard deviation computed from 7 values.

\begin{tabular}{c|cc} 
Class & Sensitivity & Specificity \\
\hline DCIS & $57 \% \pm 49$ & $99.6 \% \pm 0.6$ \\
$\begin{array}{c}\text { Excluded } \\
\text { regions }\end{array}$ & $85 \% \pm 3$ & $67 \% \pm 13$ \\
Stroma & $31 \% \pm 10$ & $97 \% \pm 2$ \\
$\begin{array}{c}\text { Solid } \\
\text { formations }\end{array}$ & $44 \% \pm 32$ & $96 \% \pm 3$ \\
$\begin{array}{c}\text { Nonneoplastic } \\
\text { objects }\end{array}$ & $71 \% \pm 45$ & $99.8 \% \pm 0.2$ \\
$\begin{array}{c}\text { Diffuse } \\
\text { invasion }\end{array}$ & $47 \% \pm 15$ & $89 \% \pm 4$
\end{tabular}

\section{CONCLUSION AND FUTURE WORK}

The recent advent of whole slides images is a great opportunity to provide new diagnostic tools and to help pathologists in their clinical analyses. However, it also comes with great challenges, mainly due to the large size of the images and the complexity of their content. To achieve a fast and efficient classification of the images, we proposed in this paper a methodology enabling to partition the initial image in relevant regions. This approach is based on an original segmentation algorithm and on a supervised classification using a textural characterization of each region. We carried out experiments on 7 annotated images and obtained promising results. In the future, we are planning to analyze the images at a lower level in order to detect each single cell and complex biological structures present in the images. We believe that identifying theses structures and being able to accurately classify them is the key to the development of future clinical tools. Furthermore, it will help to improve the development of patient-specific targeted treatment.

\section{REFERENCES}

Elston, C. W. and Ellis, I. O. (1991). Pathological prognostic factors in breast cancer. i. the value of histological grade in breast cancer: Experience from a large study with long-term follow-up. Histopathology.

Fawcett, T. (2006). An introduction to roc analysis. Pattern Recognition Letters.

Ghaznavi, F., Evans, A., Madabhushi, A., and Feldman, M. (2013). Digital imaging in pathology: Whole-slide imaging and beyond. Annual Review of Pathology.

Gurcan, M. N., Boucheron, L. E., Can, A., Madabhushi, A., Rajpoot, N. M., and Yener, B. (2009). Histopathological image analysis: a review. IEEE Reviews in Biomedical Engineering.

Homeyer, A., Schenk, A., Arlt, J., Dahmen, U., Dirsch, O., and Hahn, H. K. (2013). Practical quantification of necrosis in histological whole-slide images. Computerized Medical Imaging and Graphics. 
Huang, C.-H., Veillard, A., Roux, L., Loménie, N., and Racoceanu, D. (2010). Time-efficient sparse analysis of histopathological whole slide images. Computerized Medical Imaging and Graphics.

Montanari, U. (1971). On the optimal detection of curves in noisy pictures. Communications of the ACM.

Ojala, T., Pietikäinen, M., and Harwood, D. (1996). A comparative study of texture measures with classification based on featured distributions. Pattern Recognition.

Roullier, V., Lézoray, O., Ta, V.-T., and Elmoataz, A. (2011). Multi-resolution graph-based analysis of histopathological whole slide images: application to mitotic cell extraction and visualization. Computerized Medical Imaging and Graphics.

Ruiz, A., Sertel, O., Ujaldon, M., Catalyurek, U., Saltz, J., and Gurcan, M. (2007). Pathological image analysis using the gpu: Stroma classification for neuroblastoma. 2007 IEEE International Conference on Bioin- formatics and Biomedicine (BIBM 2007).

Sertel, O., Kong, J., Shimada, H., and Catalyurek, U. (2009). Computer-aided prognosis of neuroblastoma on whole-slide images: Classification of stromal development. Pattern Recognition.

Signolle, N., Plancoulaine, B., Herlin, P., and Revenu, M. (2008). Texture-based multiscale segmentation: Application to stromal compartment characterization on ovarian carcinoma virtual slides. Image and Signal Processing.

Tavassoli, F. A. and Devilee, P. (2003). Pathology and Genetics of Tumours of the Breast and Female Genital Organs. IARCPress.

Wemmert, C., Krüger, J., Forestier, G., Sternberger, L., Feuerhake, F., and Gançarski, P. (2013). Stain unmixing in brightfield multiplexed immunohistochemistry. IEEE International Conference on Image Processing. 